\title{
CENTIBOTS: VERY LARGE SCALE DISTRIBUTED ROBOTIC TEAMS
}

${ }^{1}$ Kurt Konolige, Charlie Ortiz, Regis Vincent, Benoit Morisset, Andrew Agno, Michael Eriksen

${ }^{2}$ Dieter Fox, Benson Limketkai, Jonathan Ko, Benjamin Stewart, Dirk Schulz

${ }^{I}$ SRI International, USA $;{ }^{2}$ University of Washington, USA

We describe the development of Centibots, a framework for very large teams of robots that are able to perceive, explore, plan and collaborate in unknown environments.

Centibots teams currently consist of approximately 100 robots, which can be deployed in unexplored areas and which can efficiently distribute tasks among themselves; the system also makes use of a mixed initiative mode of interaction in which a user can easily influence missions as necessary.

In contrast to simulation-based systems which abstract away aspects of the environment for the purposes of exploring component technologies, the Centibots design reflects an integrated end-to-end system. 